\title{
Striatal Morphology is Associated with Tobacco Cigarette Craving
}

\author{
Amy C Janes*,', Min Tae M Park², Stacey Farmer' and M Mallar Chakravarty ${ }^{2,3}$ \\ 'McLean Imaging Center, McLean Hospital, Harvard Medical School, Belmont, MA, USA; ${ }^{2}$ Cerebral Imaging Centre, Douglas Mental Health \\ University Institute, Montreal, QC, Canada; ${ }^{3}$ Department of Psychiatry, McGill University, Montreal, QC, Canada
}

\begin{abstract}
The striatum has a clear role in addictive disorders and is involved in drug-related craving. Recently, enhanced striatal volume was associated with greater lifetime nicotine exposure, suggesting a bridge between striatal function and structural phenotypes. To assess this link between striatal structure and function, we evaluated the relationship between striatal morphology and this brain region's wellestablished role in craving. In tobacco smokers, we assessed striatal volume, surface area, and shape using a new segmentation methodology coupled with local shape indices. Striatal morphology was then related with two measures of craving: state-based craving, assessed by the brief questionnaire of smoking urges (QSU), and craving induced by smoking-related images. A positive association was found between left striatal volume and surface area with both measures of craving. A more specific relationship was found between both craving measures and the dorsal, but not in ventral striatum. Evaluating dorsal striatal subregions showed a single relationship between the caudate and QSU. Although cue-induced craving and the QSU were both associated with enlarged striatal volume and surface area, these measures were differentially associated with global or more local striatal volumes. We also report a connection between greater right striatal shape deformations and cue-induced craving. Shape deformations associated with cue-induced craving were specific to striatal subregions involved in habitual responding to rewarding stimuli, which is relevant given the habitual nature of cue-induced craving. The current findings confirm a relationship between striatal function and morphology and suggest that variation in striatal morphology may be a biomarker for craving severity.

Neuropsychopharmacology (20I5) 40,406-4II; doi:I0.1038/npp.2014.185; published online 20 August 20I4
\end{abstract}

\section{INTRODUCTION}

The connection between striatal function and addiction is well founded as this brain region has a major role in subjective drug craving (Volkow et al, 2006) and the maintenance of drug-cue associations (See et al, 2007). In relation to tobacco smoking specifically, the striatum mediates the rewarding effect of smoking (Barrett et al, 2004), the motivation to smoke (Le Foll et al, 2014), and damage to this region due to stroke disrupts nicotine dependence (Gaznick et al, 2014). The striatum also shows persistent reactivity to smoking-related cues even during abstinence, which may contribute to relapse risk during a quit attempt (Janes et al, 2009; McClernon et al, 2009). Recently, larger striatal volume has been linked with greater lifetime exposure to nicotine (Das et al, 2011), which calls into question whether there is a link between striatal function and structural phenotype. Although previous studies have evaluated striatal volume in the context of addiction (Churchwell et al, 2012; Das et al, 2011; Ersche

*Correspondence: Dr AC Janes, Brain Imaging Center, Mail Stop 310, McLean Hospital, II5 Mill Street, Belmont, MA 02478, USA, Tel: +| 617855 3244, Fax: + | 6178552770 ,

E-mail: ajanes@mclean.harvard.edu

Received 2I May 20 I4; revised 27 June 20।4; accepted I5 July 20।4; accepted article preview online 24 July 2014 et al, 2012), anomalies in subcortical shape are beginning to emerge as a robust endophenotype in neuropsychiatric disorders (Shaw et al, 2014), suggesting that this measure may provide additional insight into the relationship between striatal morphology and substance abuse.

To assess the link between craving and striatal anatomy, we used a new segmentation methodology coupled with local shape indices along the striatal surface to estimate striatal volume (Chakravarty et al, 2013), surface area, and shape (Raznahan et al, 2014). For volumetric analyses, we assessed both total striatal volume as well as striatal subregions to get a sense of both global and localized structural effects. These measures provide a comprehensive evaluation of striatal morphology not easily captured by standard volumetric analyses alone. We then assessed the link between striatal morphology and two measures of cigarette craving: baseline subjective craving as measured by the brief questionnaire of smoking urges (QSU; Cox et al, 2001) and craving induced by exposure to smoking-related cues (Janes et al, 2013). Although both measures evaluate craving, the QSU assesses craving state prior to exposure to smoking cues, whereas cue-induced craving measures the amount of craving induced by visual stimuli associated with nicotine reward. We hypothesize that smokers who report more intense cigarette craving will have larger striatal volume, which may partly explain the connection between 
larger striatal volume and lifetime cigarette use (Das et al, 2011). In addition, we will build upon the existent literature by providing the first analysis that links cigarette craving and more nuanced measures of striatal morphology including surface area and shape assessments.

\section{MATERIALS AND METHODS}

\section{Participants}

Twenty-five nicotine-dependent participants (6 left-handed, 15 women/10 men) participated in all study measures at the McLean Imaging Center of McLean Hospital. Participants were $26.3 \pm 5.3$ (mean $\pm S D$ ) years old and had an average of $6.4 \pm 4.1$ pack-years of smoking experience. Pack-years of smoking use were calculated by multiplying the average number of packs of cigarettes smoked per day by the number of years the participant smoked. Inclusion criteria included smoking $\geqslant 10$ cigarettes/day over the past 6 months and on average, participants smoked $14.2 \pm 3.7$ cigarettes per day. All participants were moderately to heavily nicotine dependent as indicated by an average Fagerstrom test for nicotine dependence (Heatherton et al, 1991) score of $6.12 \pm 1.1$. Participants were assessed by the Structured Clinical Interview for DSM and met criteria for current nicotine dependence. Participants had an average expired carbon monoxide level of $24.4 \pm 12.4 \mathrm{ppm}$ and $15 \pm 2.1$ years of education. Participants were excluded if they had a lifetime diagnosis of the following conditions: organic mental disorder, bipolar depression, or schizophrenia spectrum disorder or depressive symptoms in the past 6 months. Participants were excluded for current substance use disorder other than nicotine dependence. Smokers also were excluded for pregnancy, recent drug use, or excessive alcohol use. Participants were recruited using online advertisements and fliers posted in the Boston metropolitan area. All participants provided both verbal and written informed consent prior to participating in the study and the institutional review board at McLean Hospital approved this study.

\section{Magnetic Resonance Imaging}

All participants smoked one of their own cigarettes immediately following signing the informed consent to standardize the time as a cigarette was last smoked. MRI scanning began $\sim 1.5 \mathrm{~h}$ after smoking this cigarette. Scans were acquired on a Siemens Trio 3 Tesla scanner (Erlangen, Germany) with a 32-channel head coil. Multiecho multiplanar rapidly acquired gradient-echo (ME-MPRAGE) structural images were acquired with the following parameters $(\mathrm{TR}=2.1 \mathrm{~s}, \mathrm{TE} 3.3 \mathrm{~ms}$, slices $=128$, matrix $=256$ $\times 256$, flip angle $=7^{\circ}$, resolution $=1.0 \mathrm{~mm} \times 1.0 \mathrm{~mm} \times 1.33$ $\mathrm{mm})$.

\section{Craving Measures}

Craving was assessed just prior to scanning ( $1.5 \mathrm{~h}$ after smoking) using the 10-item QSU (Cox et al, 2001), which is a standard tobacco craving measure. Following scanning ( $3 \mathrm{~h}$ after smoking), participants were shown smoking and neutral images and were asked to rate the amount of craving they experienced when viewing each image on a five-point scale. Smoking and neutral images were comprised of images from various sources including the International Smoking Image Series (Gilbert and Rabinovich, 1999) and those used in our prior work (Janes et al, 2013) and by Kober et al (2010). To control for visual characteristics, all images were converted to gray scale. The smoking images represented people smoking, hands holding cigarettes, and items such as cigarettes. The neutral images, which lacked the smoking element, were matched for content and included images of people, hands holding objects such as paintbrushes, and items such as pens. One hundred and forty-four images of each type were presented. The average difference in craving to smoking $v s$ neutral images was then calculated and was associated with brain morphology. Average reported subjective craving between smoking and neutral images was compared using a paired $t$-test.

\section{Striatal Volume and Surface Area}

Neuroanatomical segmentations are typically limited in accuracy by errors in the transformation estimation, irreconcilable neuroanatomical differences between the neuroanatomy of the template and the subject, and resampling errors after the application of the transformation to the labels (Collins and Pruessner, 2010; Heckemann et al, 2006). MAGeT Brain limits these errors by using a modified 'multiatlas'-based strategy while still relying on a single welldefined template. As input, the algorithm uses a single atlas containing the striatum, which was previously defined using a three-dimensional reconstruction of serial histological data (Chakravarty et al, 2006) that was warped to an MRI template (Holmes et al, 1998). The atlas is first customized to a subset of the data set (21 subjects, based on an arbitrary subsample of the cohort being studied) using a nonlinear transformation estimated in a region of interest defined around the subcortical structures (Chakravarty et al, 2008, 2009, 2012). This set of subjects now acts as a set of templates to which all other subjects are now warped, providing 21 candidate segmentations for each subject and effectively uses the neuroanatomical variability of the cohort being analyzed to improve overall segmentation accuracy. The final segmentation is decided upon by using a voxel-wise majority vote (ie, the label occurring most frequently at a specific voxel location is retained; Collins et al, 1995). In our original paper, we demonstrated high overlap between our method and gold standards manually derived on MRI data (Dice Kappa of 0.861 when using MAGeT Brain with the ANIMAL algorithm (Collins et al, 1995) for nonlinear transformation estimation; Chakravarty et al, 2013). As in our recent work (Raznahan et al, 2014), we also quantified local surface area. A surface-based model was warped to each subject using all 21 transformation estimated through the MAGeT Brain segmentation procedure. To ensure homology between vertices, all surface vertices were redefined using a Vornoi diagram (see Lyttleton et al, 2009). The median position was taken for each coordinate at each vertex in order to create a 'median surface' representation. This can be considered to be the surface-based homolog to majority-vote label fusion measures and has the benefit of filtering noise and outliers from the surface-based representation. Total surface area was 
then estimated as the sum of the surface area for each polygon in the surface. If a significant relationship between craving and total striatal morphology was identified, a striatal subregion analysis was then conducted to identify a more localized effect. Striatal subregion volumes were estimated using the MAGeT Brain algorithm as well. In this case, the striatal mask was subdivided in the original histological data set in order to identify the ventral striatum and the caudate and putamen.

\section{Vertex-Wise Analyses of Striatal Shape: Surface Displacement}

Shape analysis was carried out using a surface-based methodology proposed by Lerch et al (2008). First, surface-based representations of the striatum defined on the input atlas were estimated using the marching cubes algorithm (Lorensen and Cline, 1987) and morphologically smoothed using the AMIRA software package (Visage Imaging, San Diego, CA, USA). The resulting surfaces are defined by $\sim 6300$ vertices per striatum. The nonlinear portions of the 21 transformations that map each subject to the input atlas were concatenated and then averaged across the template library in order to limit the effects of noise and error and to increase precision and accuracy (Frey et al, 2011; Dorr et al, 2008; Borghammer et al, 2010). The dot product between the nonlinear deformation vector (of the inverse of the averaged atlas-to-subject transformation) and the striatum surface normal (a unit vector describing the direction perpendicular to the surface) is estimated and provides a local measure of inward or outward displacement along the normal (as first described in Lerch et al (2008); and as in the method described in Magon et al, (2014)). Prior to estimating the dot product between the normal to the surface and the deformation field, we explicitly modeled and removed global linear effects not originally accounted for in the initial linear transformations. This ensures that the contributions of overall differences in brain volume are minimized in this analysis.

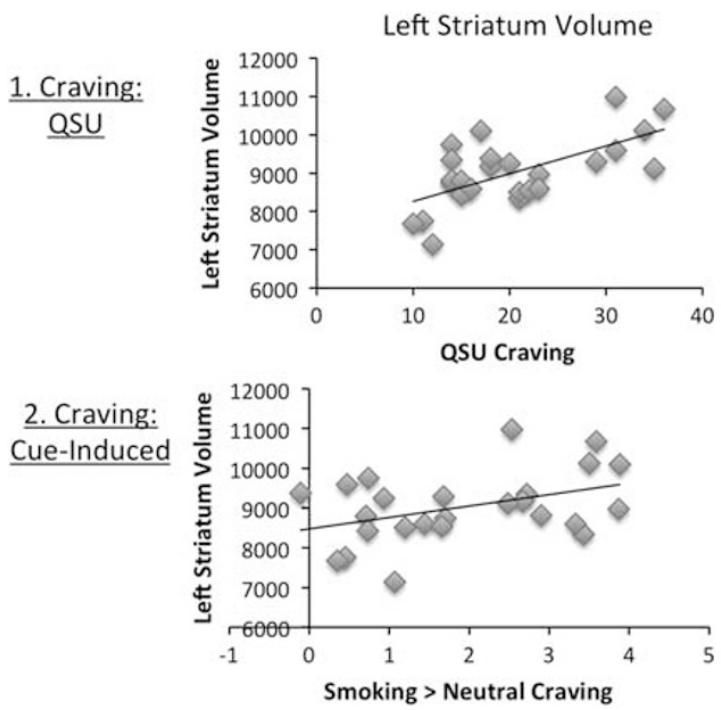

\section{Statistics}

RMINC (https://github.com/mcvaneede/RMINC), a statistical image analysis software package built to work in the $\mathrm{R}$ environment, was used for all surface-based vertex-wise analyses. A linear model was used and all imaging results were corrected using a $10 \%$ false discovery rate (FDR) correction (Genovese et al, 2002). All analyses with respect to PSYRATS subscales were conducted using the values of the assessment. SPSS was used to conduct correlation and regression analyses. Correlations were conducted to evaluate the relationship between QSU and cue-induced craving measures, whereas regression analyses were used to evaluate associations between brain morphology and craving measures. For the volumetric analyses, total brain volume, age, and sex were included as control variables.

\section{RESULTS}

\section{Subjective Craving and Smoking History}

Participants reported experiencing significantly greater craving when viewing smoking $v s$ neutral images $(t=7.6$, $p<0.0001)$. Smoking $v s$ neutral cue-induced craving was positively correlated with craving as measured by the QSU $(r=0.42, p=0.035)$.

\section{Striatal Morphology}

Striatal volume. A significant positive association was found between left striatal volume and craving as measured by the QSU ( $t=2.6, p=0.016$; Figure 1$)$ and craving induced by exposure to smoking $v s$ neutral cues $(t=2.3$, $p=0.032$; Figure 1). Given this significant association, a follow-up subregion analysis was conducted. Dorsal, but not ventral, striatal volume was related with both the QSU $(t=2.3, p=0.026)$ and smoking $v s$ neutral cue-induced craving $(t=2.3, p=0.03)$. Craving measured by the QSU was significantly associated with the caudate $(t=3.3$, $p=0.004)$, but not the putamen. Smoking $v s$ neutral cueinduced craving was not related to either dorsal striatal

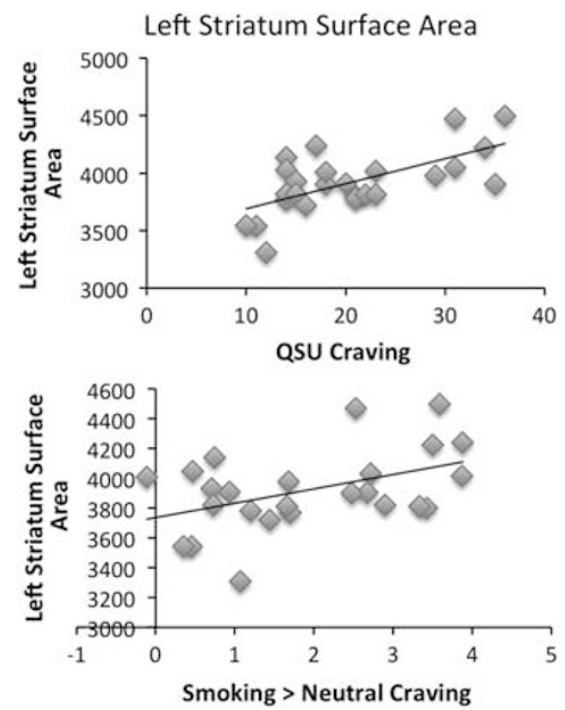

Figure I Left striatal volume and surface area relationships with craving. A positive relationship was found between craving ( I. QSU and 2. cue-induced) and left striatal volume and surface area. 
subregion. No significant associations were found between right total striatal volume and either craving measure.

Surface area. A positive association was found between the left striatal surface area QSU $(t=2.5, p=0.02$; Figure 1) and smoking $v s$ neutral cue-induced craving $(t=0.02$, $p=0.007$, Figure 1).

Striatal shape. A significant relationship was found between smoking $v s$ neutral cue-induced craving and right striatal shape deformations for both local contractions $(t$-statistic range: $-2.7<t<-3.95)$ and expansions $(t$-statistic range: $2<t<3.56) \quad$ (FDR corrected $10 \%$; Figure 2). Similar trends were found for the left striatum, but were not significant (Figure 2). No relationship was found between striatal shape and QSU.

\section{DISCUSSION}

These current findings show a relationship between cigarette craving and striatal morphology. Both subjective craving, as measured by the QSU, and craving induced by exposure to smoking cues were associated with larger left striatal volume and surface area. Cue-induced craving was significantly related with more profound right striatal shape deformations. Given the striatum's functional role in craving (Volkow et al, 2006), and cue-reactivity (See et al, 2007) the current findings suggest a link between striatal morphology and its underlying function. In addition, our novel surface area and shape findings provide more nuanced insight into the relationship between striatal morphology and craving.

The relationship between craving and left lateralized striatal volume and surface area is consistent with previous findings linking stimulant abuse with greater left striatal volume (Das et al, 2011; Churchwell et al, 2012, Ersche et al, 2012). The connection between the left striatum and
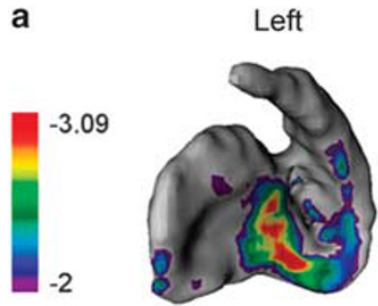

b
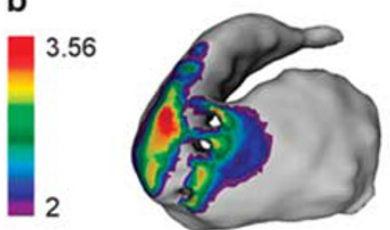

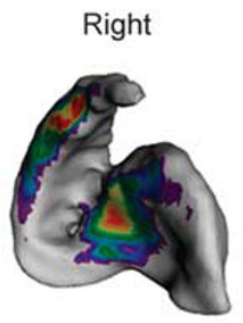

$-3.95$

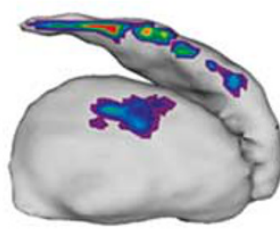

substance abuse may be tied to the more dominant role of the left hemisphere in reward and approach behavior (Davidson and Irwin, 1999). In fact, the left striatum shows greater activation following unexpected reward delivery (McClure et al, 2003) and sustained activation following reward feedback (Delgado et al, 2000). Greater positive incentive motivation also is associated with higher dopamine $\mathrm{D} 2$ receptor availability in the left relative to the right striatum (Tomer et al, 2008). It is tempting to speculate that the relationship between left striatal volume and craving is due to greater left striatal D2 receptor availability. Such a hypothesis is plausible given that D2 binding potential is positively associated with striatal volume (Woodward et al, 2009), and craving (Volkow et al, 2006).

When assessing more localized left striatal subregions, both the QSU and smoking cue-induced craving measures were associated with the dorsal, but not ventral striatum. On further inspection, only the QSU was associated with more localized volume in the caudate, indicating that the specificity of the volumetric association depends on the type of craving evaluated. This specific relationship between caudate volume and QSU is consistent with the caudate's role in craving (Volkow et al, 2006) and reward processing (Delgado et al, 2000). In contrast, smoking cue-induced craving is associated with enhanced dorsal striatal volume, which includes both the caudate and putamen. These findings suggest that craving, induced by exposure to smoking-related cues may involve more distributed striatal structures, whereas subjective craving, independent of cue-exposure, is associated with more localized caudate morphology.

A relationship between smoking cue-induced craving and right dorsal striatal shape was found independent of overall striatal volume, specifically in the ventral anterior putamen and the right caudate tail. The ventral anterior putamen not only is larger in occasional stimulant users (Mackey et al, 2014), but this striatal subregion receives projections from the insula (Chikama et al, 1997), a brain region implicated in maintaining nicotine dependence (Naqvi et al, 2007) and smoking cue-reactivity (Janes et al, 2010). The insula and striatum may interact to mediate nicotine dependence, as damage to both of these brain regions disrupts smoking (Gaznick et al, 2014), and suggests a possible link between ventral anterior putamen deformations and cue-reactivity due to striatal-insula interactions. The fact that cuereactivity was associated with shape differences in the caudate tail is particularly interesting given the role of this striatal subregion in visual habits (Fernandez-Ruiz et al, 2001). This is relevant to the current findings owing to the habitual nature of smoking cue-reactivity (Everitt et al, 2001). The caudate tail facilitates visual habits as this striatal subregion interacts with inferior temporal brain areas as part of the 'visual' corticostriatal loop, thus providing a 'link in a visual pathway underlying oculomotor control and visuo-motor associations' (Saint-Cyr et al, 1990, pg.1). Owing to this link, the caudate tail facilitates automatic responding toward environmental stimuli, which have been consistently rewarded (Yamamoto et al, 2013). The caudate tail most likely maintains the automatic and habitual responding to the smoking-related images used in the current study, as these images are 'consistently rewarded visual stimuli' owing to their repeated pairing with the reinforcing experience of nicotine. As subcortical shape
Figure 2 Cue-induced craving and striatal shape deformations. A significant association was found between smoking cue-induced craving and both local (a) contractions and (b) expansions in the right striatum. Colored regions indicate the range of $t$-statistics and regions of significance after FDR correction $(q=0.1)$, only applicable for the right striatum. An arbitrary threshold $(t=2)$ is chosen for trend-level results for the left striatum, which are not significant after correction. 
measures are beginning to emerge as a robust endophenotype in neuropsychiatric disorders (Shaw et al, 2014), it is possible that individuals with the variability in striatal shape, we report, might be predisposed to form stronger visual habits. However, this possibility is speculative and requires additional testing.

Although the current work shows a clear link between striatal morphology and craving, additional research will aid in our understanding of how striatal morphology contributes to addiction and other habitual behaviors. For instance, future studies should consider different populations of smokers, as we restricted our sample to a relatively young, healthy population. Whether variation in striatal morphology continues to be associated with craving following brain changes associated with aging remains to be determined. Whereas our sample size is comparable to prior anatomical studies of nicotine dependence (Brody et al, 2004), research involving larger sample sizes may address some of these additional questions. In addition, the link between craving and striatal morphology prompts the question of whether variation in striatal morphology could be linked with differences in other 'visual habits' relevant to normal learning or other forms of craving and compulsive disorders. For example, enhanced striatal surface area has been associated with obsessive-compulsive disorder (OCD; Shaw et al, 2014), suggesting that larger striatal surface area may be a feature of compulsive disorders including OCD and addiction. Finally, our research focused only on the relationship between striatal morphology and cigarette craving as previous research has shown a clear link between stimulant abuse and enhanced striatal volume when substance abusers and healthy controls were compared (Das et al, 2011; Churchwell et al, 2012, Ersche et al, 2012). It is unclear whether variability in striatal morphology, in non-abusing populations also may explain individual differences in craving severity for other, more natural, reinforcers. Collectively, our findings indicate that striatal morphology is related to this brain regions' role in craving and that morphological assessment of the striatum, beyond standard volumetric analysis, provides more nuanced information regarding the relationship between striatal morphology and function.

\section{FUNDING AND DISCLOSURE}

This work was supported by the National Institute on Drug Abuse Grant K01DA029645 (AJ). The other authors declare no conflict of interest.

\section{REFERENCES}

Barrett SP, Boileau I, Okker J, Pihl RO, Dagher A (2004). The hedonic response to cigarette smoking is proportional to dopamine release in the human striatum as measured by positron emission tomography and $[11 \mathrm{C}]$ raclopride. Synapse 54: 65-71.

Borghammer P, Ostergaard K, Cumming P, Gjedde A, Rodell A, Hall N et al (2010). A deformation-based morphometry study of patients with early-stage Parkinson's disease. Eur J Neurol 17: 314-320.
Chakravarty MM, Bertrand G, Hodge CP, Sadikot AF, Collins DL (2006). The creation of a brain atlas for image guided neurosurgery using serial histological data. Neuroimage 30: 359-376.

Chakravarty MM, Broadbent S, Rosa-Neto P, Lambert CM, Collins DL (2009). Design, construction, and validation of an MRIcompatible vibrotactile stimulator intended for clinical use. J Neurosci Methods 184: 129-135.

Chakravarty MM, Felsky D, Tampakeras M, Lerch JP, Mulsant BH, Kennedy J L et al (2012). DISC1 and Striatal Volume: A Potential Risk Phenotype For mental Illness. Front Psychiatry 3: 57.

Chakravarty MM, Sadikot AF, Germann J, Bertrand G, Collins DL (2008). Towards a validation of atlas warping techniques. Med Image Anal 12: 713-726.

Chakravarty MM, Sadikot AF, Germann J, Hellier P, Bertrand G, Collins DL (2009). Comparison of piece-wise linear, linear, and nonlinear atlas-to-patient warping techniques: analysis of the labeling of subcortical nuclei for functional neurosurgical applications. Hum Brain Mapp 30: 3574-3595.

Chakravarty MM, Steadman P, van Eede MC, Clacott RD, Gu V, Shaw $\mathrm{P}$ et al (2013). Performing label-fusion-based segmentation using multiple automatically generated templates. Hum Brain Mapp 34: 2635-2654.

Chikama M, McFarland NR, Amaral DG, Haber SN (1997). Insular cortical projections to functional regions of the striatum correlate with cortical cytoarchitectonic organization in the primate. J Neurosci 17: 9686-9705.

Churchwell JC, Care PD, Ferrett HL, Stein DJ, Yurgelun-Todd DA (2012). Abnormal striatal circuitry and intensified novelty seeking among adolescents who abuse methamphetamine and cannabis. Dev Neurosci 34: 310-317.

Collins DL, Holmes CJ, Peters TM, Evans AC (1995). Automatic 3D model-based neuroanatomical segmentation. Hum Brain Mapp 3: 190-208.

Collins DL, Pruessner JC (2010). Towards accurate, automatic segmentation of the hippocampus and amygdala from MRI by augmenting ANIMAL with a template library and label fusion. Neuroimage 52: 1355-1366.

Cox LS, Tiffany ST, Christen AG (2001). Evaluation of the brief questionnaire of smoking urges (QSU-brief). in laboratory and clinical settings. Nicotine Tob Res 3: 7-16.

Das D, Cherbuin N, Anstey KJ, Sachdev PS, Easteal S (2011). Lifetime cigarette smoking is associated with striatal volume measures. Addict Biol 17: 817-825.

Davidson RJ, Irwin W (1999). The functional neuroanatomy of emotion and affective style. Trends Cog Sci 3: 11-21.

Delgado MR, Nystrom LE, Fissell C, Noll DC, Fiex JA (2000). Tracking the hemodynamic responses to reward and punishment in the striatum. J Neurophysiol 84: 3072-3077.

Dorr AE, Lerch JP, Spring S, Kabani N, Henkelman RM (2008). High resolution three-dimensional brain atlas using an average magnetic resonance image of 40 adult C57Bl/6J mice. Neuroimage 42: 60-69.

Ersche KD, Jones PS, Williams GB, Turton AJ, Robbins TW, Bullmore ET (2012). Abnormal brain structure implicated in stimulant drug addiction. Science 335: 601-604.

Everitt BJ, Dickinson A, Robbins TW (2001). The neuropsychological basis of addictive behavior. Brain Res Rev 36: 129-138.

Fernandez-Ruiz J, Wang J, Aigner TG, Mishkin M (2001). Visual habit formation in monkeys with neurotoxic lesions of the ventrocaudal neostriatum. Proc Natl Acad Sci USA 98: 4196-4201.

Frey S, Pandya DN, Chakravarty MM, Bailey L, Petrides M, Collins DL (2011). An MRI based average macaque monkey stereotaxic atlas and space (MNI monkey space). Neuroimage 55: 1435-1442.

Gaznick N, Tranel D, McNutt A, Bechara A (2014). Basal ganglia plus insula damage yields stronger disruption of smoking addiction than basal ganglia damage alone. Nicotine Tob Res 16: $445-453$. 
Genovese CR, Lazar NA, Nichols T (2002). Thresholding of statistcal maps in functional neuroimaging using the false discovery rate. Neuroimage 15: $870-878$.

Gilbert DG, Rabinovich NE (1999). International smoking image series (with neutral counterparts), Version 1.2. Integrative Neuroscience Laboratory, Department of Psychology, Southern Illinois University: Carbondale, IL, USA.

Heatherton TF, Kozlowski LT, Frecker RC, Fagerstrom KO (1991). The Fagerstrom Test For Nicotine Dependence: A revision of the Fagerstrom Tolerance Questionnaire. $\mathrm{Br} J$ Addict 86: 1119-1127.

Heckemann RA, Hajnal JV, Aljabar P, Rueckert D, Hammers A (2006). Automatic anatomical brain MRI segmentation combining label propagation and decision fusion. Neuroimage 33: 115-126.

Holmes CJ, Hoge R, Collins L, Woods R, Toga AW, Evans AC (1998). Enhancement of MR images using registration for signal averaging. J Comput Assist Tomorgr 22: 324-333.

Janes AC, Frederick BB, Richardt S, Burbridge C, Merlo-Pich E, Reshaw PF et al (2009). Brain fMRI reactivity to smoking-related images before and during extended smoking abstinence. Exp Clin Psychopharmacol 17: 365-373.

Janes AC, Pizzagalli DA, Richardt S, Frederick BB, Chuzi S, Pachas $\mathrm{G}$ et al (2010). Brain reactivity to smoking cues prior to smoking cessation predicts ability to maintain tobacco abstinence. Biol Psychiatry 67: 722-729.

Janes AC, Ross RS, Farmer S, Frederick BB, Nickerson LD, Lukas SE et al (2013). Memory retrieval of smoking-related images induce greater insula activation as revealed by an fMRI-based delayed matching to sample task. Addict Biol (e-pub ahead of print 22 November 2013; doi:10.111/abd.12112).

Kober H, Mende-Siedlecki P, Kross EF, Weber J, Mischel W, Hart CL et al (2010). Prefrontal-striatal pathway underlies cognitive regulation of craving. Proc Natl Acad Sci USA 107: 14811-14816.

Le Foll B, Guranda M, Wilson AA, Houle S, Rusjan PM, Wing VC et al (2014). Elevation of dopamine induced by cigarette smoking: novel insights from a [(11).C]-(+).-PHNO PET study in humans. Neuropsychopharmacology 39: 415-424.

Lerch JP, Carroll JB, Spring S, Bertram LN, Schwab C, Hayden MR et al (2008). Automated deformation analysis in the YAC128 Huntington disease mouse model. Neuroimage 39: 32-39.

Lorensen WE, Cline HE (1987). Marching cubes: a high resolution 3D surface construction algorithm. AMC SIGGRAPH Computer Graphics 21: 163-169.

Lyttelton OC, Karama S, Ad-Dab'bagh Y, Zatorre RJ, Carbonell F, Worsley K et al (2009). Positional and surface area asymmetry of the human cerebral cortex. Neuroimage 46: 895-903.
Mackey S, Stewart JL, Connly CG, Tapert SF, Paulus MP (2014). A voxel-based morphometry study of young occasional users of amphetamine-type stimulants and cocaine. Drug and Alcohol Depend 135: 104-111.

Magon S, Chakravarty MM, Amann M, Weier K, Maegelin Y, Andelova $\mathrm{M}$ et al (2014). Label-fusion-segmentation and deformation-based shape analysis of deep gray matter in multiple sclerosis: the impact of thalamic subnuclei on disability. Hum Brain Mapp 35: 4193-4203.

McClernon FJ, Kozink RV, Lutz AM, Rose JE (2009). 24-h smoking abstinence potentiates fMRI-BOLD activation to smoking cues in cerebral cortex and dorsal striatum. Psychopharmacology 204: 25-35.

McClure SM, Berns GS, Montague R (2003). Temporal prediction errors in a passive learning task activate human striatum. Neuron 38: 339-346.

Naqvi NH, Rudrauf D, Damasio H, Bechara A (2007). Damage to the insula disrupts addiction to cigarette smoking. Science 315: 531-534.

Raznahan A, Shaw PW, Lerch JP, Clasen LS, Greenstein D, Berman R et al (2014). Longitudinal four-dimensional mapping of subcortical anatomy in human development. Proc Natl Acad Sci USA 111: 1592-1597.

Saint-Cyr JA, Ungerleider LG, Desimone R (1990). Organization of visual cortical inputs to the striatum and subsequent outputs to the pallido-nigral complex in the monkey. J Comp Neurol 298: 129-156.

See RE, Elliott JC, Feltenstein MW (2007). The role of dorsal vs ventral striatal pathways in cocaine-seeking behavior after prolonged abstinence in rats. Psychopharmacology 194: 321-331.

Shaw P, Sharp W, Sudre G, Wharton A, Greenstein D, Raznahan A et al (2014). Subcortical and cortical morphological anomalies as an endophenotype in obsessive-compulsive disorder. Mol Psychiatry e-pub ahead of print 11 February 2014 doi:10.1038/ mp.2014.3.

Tomer R, Goldstein RZ, Wang G, Wong C, Volkow ND (2008). Incentive motivation is associated with striatal dopamine asymmetry. Biol Psychology 22: 98-101.

Volkow ND, Wang G, Telang F, Fowler JS, Logan J, Childress AR et al (2006). Cocaine cues and dopamine in dorsal striatum: Mechanism of craving in cocaine addiction. J Neurosci 26: 6583-6588.

Woodward ND, Zald DH, Ding Z, Riccardi P, Ansari MS, Baldwin RM et al (2009). Cerebral morphology and dopamine D2/D3 receptor distribution in humans: a combined [18F]fallypride and voxel-based morphometry study. Neuroimage 46: 31-38.

Yamamoto S, Kim HF, Hikosaka O (2013). Reward valuecontingent changes of visual responses in primate caudate tail associated with a visuomotor skill. J Neurosci 3: 11227-11238. 